\title{
RAS mimetic revealed
}

The binding
site for
rigosertib was
mapped to the
RAS-binding
domain (RBD)
of RAF,
a conserved
domain that
is shared by
a number of
RAS effector
proteins.

The majority of human cancers exhibit aberrant signalling of the small G protein RAS. This can occur through RAS point mutations, which are oncogenic drivers in $20-30 \%$ of human cancers, or through mutations in upstream or downstream signalling molecules. However, the development of RAS inhibitors has been challenging. Now, reporting in Cell, E. Premkumar Reddy and colleagues show that the small-molecule inhibitor rigosertib acts as a RAS mimetic - by disrupting the association of RAS with RAF and other effector proteins, it inactivates RAS downstream signalling.

Rigosertib was originally developed as an inhibitor of Polo-like kinase 1 (PLK1). However, it does not bind to PLK1 directly, and it

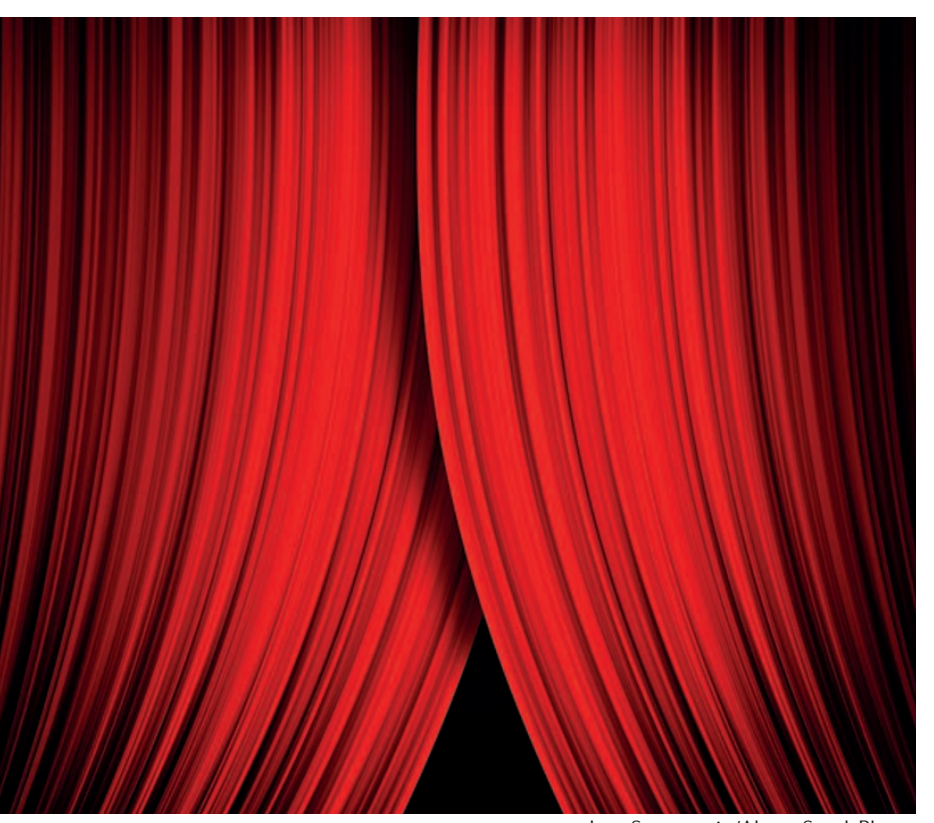

Igor Stevanovic/Alamy Stock Photo also inhibits the phosphoinositide 3-kinase (PI3K) pathway, which is commonly activated in cancer cells. The precise mechanism of action of rigosertib had not been defined.

The authors now used a pulldown assay and mass spectrometry to identify its targets. Interestingly, these included several members of the RAF family of serine/threonine kinases, which are RAS effector proteins. The binding site for rigosertib was mapped to the RAS-binding domain (RBD) of RAF, a conserved domain that is shared by a number of RAS effector proteins. Further analysis revealed that the drug can bind in two orientations, both of which occupy the very region of the RBD that is also crucial for RAS binding. Mutations in the CRAF RBD that affect RAS binding were found to have a similar effect on rigosertib binding, indicating that rigosertib acts as a RAS mimetic.

Biochemical analysis of epidermal growth-factor (EGF)-activated cells confirmed that rigosertib interferes with the ability of wild-type and mutant RAS to bind to RAF proteins, and that it inhibits RAF heterodimerization and subsequent downstream signalling through mitogen-activated protein kinase kinase (MEK) and extracellular signal-regulated kinase (ERK). Rigosertib also blocks the phosphorylation of CRAF Ser338, which is required for binding of RAF to PLK1, and inhibition of this interaction was previously shown to lead to mitotic arrest. Moreover, rigosertib was found to bind to the RBDs of other RAS effectors such as
PI3K family members, thereby inhibiting the PI3K-AKT pathway. Using fibroblasts transfected with mutant RAS or p110 $\alpha$ (a subunit of PI3Ka), or wild-type PI3K $\gamma$, PI3K $\beta$ or PI3K $\delta$, the authors showed that rigosertib can inhibit cellular transformation by each of these oncogenic proteins.

Next, mice with xenografts of human colorectal and lung cancers were treated with twice-daily intraperitoneal injections of rigosertib, leading to a significant reduction of growth and mitogen-activated protein kinase (MAPK) and PI3K signalling in the tumours of these mice compared with the tumours of vehicle-treated mice. Similar results were obtained in a genetic mouse model of pancreatic cancer.

In line with these findings, a recent Phase III trial in patients with myelodysplastic syndrome reported that patients with cytogenetic abnormalities associated with RAS activation responded best to treatment with rigosertib. Together, these data support the therapeutic value of targeting a conserved protein domain to block interactions of RAS with downstream effectors. The authors point out that this strategy may similarly be applied to other $G$ proteins, potentially paving the way for a new class of drugs for $\mathrm{G}$ protein-mediated diseases.

Alexandra Flemming This article also appears in Nat. Rev. Cancer

ORIGINAL ARTICLE Athuluri-Divakar, S.K. et al. A small molecule RAS-mimetic disrupts RAS association with effector proteins to block signaling. Cell 165, 643-655 (2016) 